LAWRENCE LIVERMORE N A T IO N A L LABORATORY
Transmitted Laser Beam Diagnostic at the Omega Laser Facility

C. Niemann, G. Antonini, S. Compton, S.H. Glenzer, D. Hargrove, J.D. Moody, R.K. Kirkwood, V. Rekow, C. Sorce, W. Armstrong, R. Bahr, R. Keck, G. Pien, W. Seka, K. Thorp

April 8, 2004

15th Topical Conference on High-Temperature Diagnostics San Diego, CA, United States April 19, 2004 through April 22, 2004 
This document was prepared as an account of work sponsored by an agency of the United States Government. Neither the United States Government nor the University of California nor any of their employees, makes any warranty, express or implied, or assumes any legal liability or responsibility for the accuracy, completeness, or usefulness of any information, apparatus, product, or process disclosed, or represents that its use would not infringe privately owned rights. Reference herein to any specific commercial product, process, or service by trade name, trademark, manufacturer, or otherwise, does not necessarily constitute or imply its endorsement, recommendation, or favoring by the United States Government or the University of California. The views and opinions of authors expressed herein do not necessarily state or reflect those of the United States Government or the University of California, and shall not be used for advertising or product endorsement purposes. 


\title{
Transmitted laser beam diagnostic at the Omega laser facility
}

\author{
C. Niemann, G. Antonini, S. Compton, S.H. Glenzer, D. \\ Hargrove, J.D. Moody, R.K. Kirkwood, V. Rekow, and C. Sorce \\ Lawrence Livermore National Laboratory, 7000 East Avenue, Livermore, CA 94550 \\ W. Armstrong, R. Bahr, R. Keck, G. Pien, W. Seka, and K. Thorp \\ Laboratory for Laser Energetics, 250 E. River Road, Rochester, NY 14623
}

\begin{abstract}
We have developed and commissioned a transmitted beam diagnostic (TBD) for the $2 \omega$ high intensity interaction beam at the Omega laser facility. The TBD consists of a bare-surface reflector mounted near the target, which collects and reflects $4 \%$ of the transmitted light to a detector assembly outside the vacuum chamber. The detector includes a time integrating near-field camera that measures beam spray, deflection and the absolute transmitted power. We present a detailed description of the instrument and the calibration method and include first measurements on laser heated gasbag targets to demonstrate the performance of the diagnostic.
\end{abstract}

\section{INTRODUCTION}

Understanding laser-beam transmission through large scale-length laser produced plasmas is crucial for indirect drive inertial confinement fusion experiments. Plasma induced beam deflection and spray due to self-focusing and filamentation as well as beam absorption by parametric instabilities alter the beam intensity distribution at the fusion target and can have important effects on capsule symmetry and fusion yield $[1,2]$.

We have developed and commissioned a transmitted beam diagnostic $(\mathrm{TBD})$ for the high intensity $\left(\sim 10^{15}\right.$ $\left.\mathrm{W} / \mathrm{cm}^{2}\right) 2 \omega(527 \mathrm{~nm})$ interaction beam [3] at the Omega laser facility [4] to study $2 \omega$ beam transmission in fusion relevant plasmas. The increased laser damage threshold at $2 \omega$ compared to $3 \omega$ allows us to place collection optics close to target chamber center (tcc), which reflects transmitted light to a detector assembly outside the target chamber. This design makes the diagnostic very flexible and expandable compared to previous instruments for $3 \omega$ laser light [5], because the diagnostics components in the vacuum chamber are mounted on a standard retractable cart .

\section{TRANSMITTED BEAM DIAGNOSTIC}

The optical mirror that collects and reflects transmitted light to the detector assembly is mounted on a mechanical arm in one of the target insertion modules (TIM 1 ). The arm and its associated support structure is installed in a TIM-boat that can be extended into the target chamber (Fig. 1). This is a three step process which is controlled by a programmable logic controller (PLC) which monitors the TIM boat insertion depth. Upon initial insertion the TIM boat is halted at a predefined depth. A PLC controlled powersupply then energizes the TBD motor brake which allows the spring loaded swing arm to deploy. An electrical contact limit switch at a hard-stop defines the final opening angle. Once the arm is deployed, the TIM-boat is extended further into the

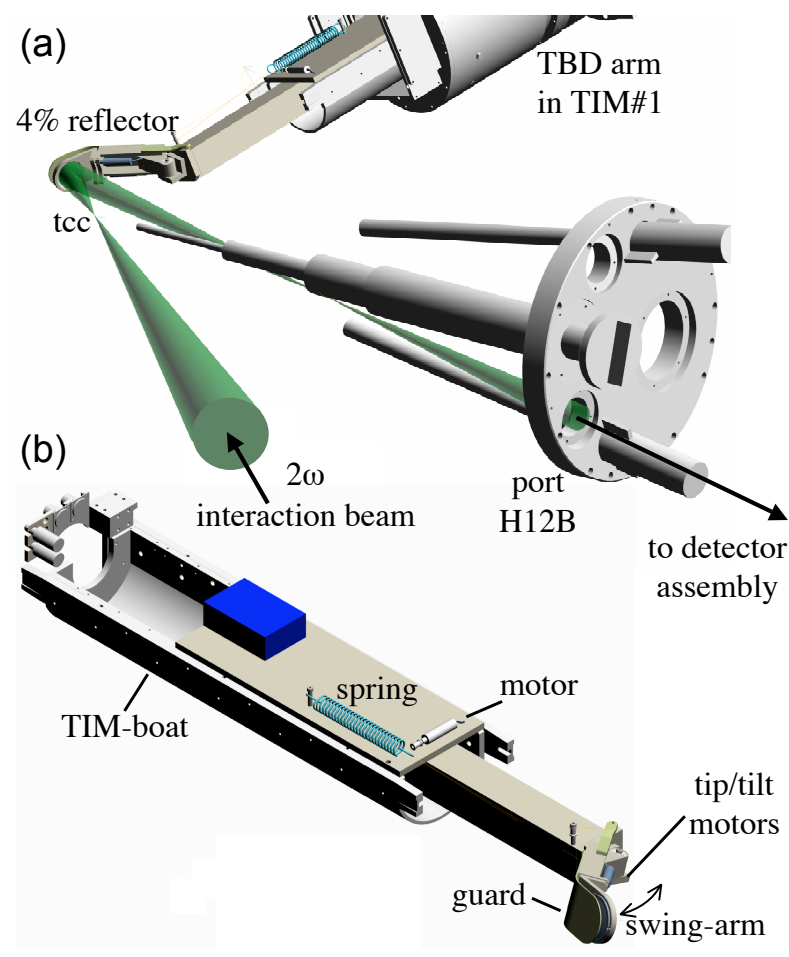

FIG. 1: (a) Setup of the TBD with the $4 \%$ reflector that reflects transmitted light out of the chamber. (b) The mechanical arm in a TIM-boat is deployed by a spring and retracted with a motor.

chamber until the mirror is in its final position, $23 \mathrm{~cm}$ from tcc. This procedure is necessary since the swing arm would interfere with other diagnostics when opening at its final position and the TIM boat is too small to accommodate an arm that is already deployed before entering the chamber. Even with this procedure the minimum clearance to other diagnostics is only $1.4 \mathrm{~cm}$, while the nearest beam cones of the 58 Omega-laser beams are $4.4 \mathrm{~cm}$ from the arm assembly in its final position. When removing the mirror from the chamber a motor spools a wire to retract the arm before it enters the TIM-boat. A 
guard provides a fail-safe method to retract the arm in the case the spool motor loses power (Fig. 1b). Although the angle of the mirror manipulator in its final position has been designed to reflect the $2 \omega$ high intensity interaction beam exactly through a port in the Omega target chamber (H12B), the arm-assembly is equipped with two remote controlled kinematic mirror motors $\left(\right.$ Oriel $^{\mathrm{TM}} \mathrm{Mo}-$ tor Mikes) to provide tip/tilt fine adjustment capability.

The 3" diameter spherical fused silica bare-surface mirror collects and reflects $4 \%$ of the transmitted light over twice the initial $\mathrm{f} / 6.7$ cone of the beam through a port window to the detector assembly outside the vacuum chamber. The mirror has a high-intensity laser antireflex (AR) coating for $2 \omega$ on the flat back-side. The uncoated concave front-side $(\mathrm{R}=37 \mathrm{~cm})$ reflects the divergent beam behind the target as a convergent beam towards the chamber port, producing a focus inside the vacuum chamber and a divergent $\mathrm{f} / 13$ beam with a diameter of $3.8 \mathrm{~cm}$ at the port window (Fig. 2).

An aspheric lens $(\mathrm{f}=80 \mathrm{~cm})$ images the TBD collection mirror plane onto a Lambertian diffuser plate (Spectralon $^{\mathrm{TM}}$ ) with an optical magnification of 1.7:1. A second $4 \%$ splitter reflects a small fraction of the beam onto the diffuser plate, while the remaining beam energy is measured in a full-aperture calorimeter (Fig. 2). The 2" calorimeter is filtered for $2 \omega$ using a combination

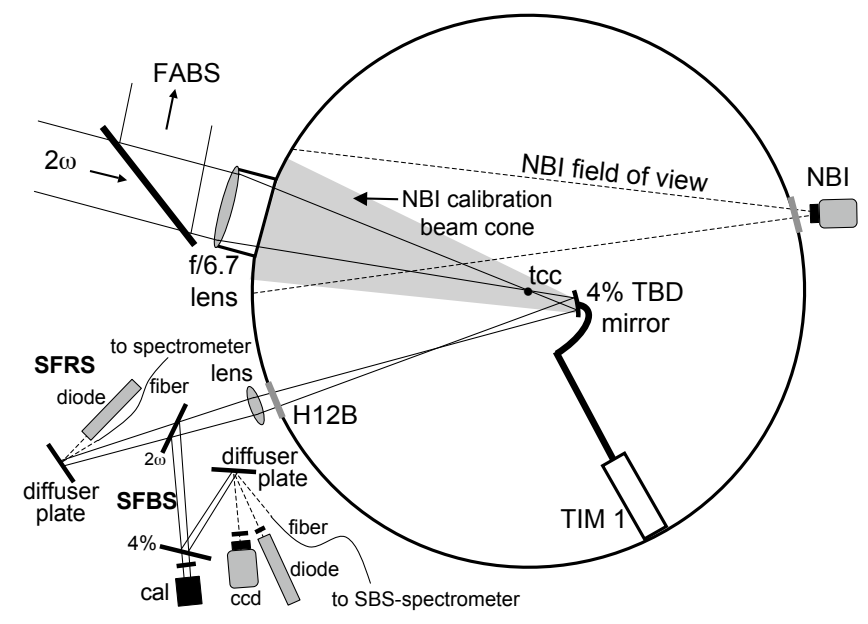

FIG. 2: Schematic of the TBD and the detector, including also the optical fibers (OF) and the second photo-diode for the TBD upgrade (section IV).

of BG18 and CS3-144 glass to reject both unconverted $1 \omega$ and $3 \omega$ laser light from the heater beams. Time integrated two-dimensional near-field images of the transmitted light on the diffuser plate are recorded with an 8-bit CCD camera (Pulnix TM-1020-15CL) through a $2 \omega$ bandpass filter (10 nm FWHM), imaging the laser spot on the diffuser plate.

The images show the intensity distribution on the mirror behind the target over twice the initial $\mathrm{f} / 6.7$ cone of the interaction beam $\left(8.5^{\circ}\right.$ around the beamaxis) with a resolution of $0.15^{\circ}$. Beam spray and deflection can directly be derived from those images.

The temporal pulse-shape of the transmitted light is recorded with a fast photo-detector (Hamamatsu R1328U-03, 60 ps rise time) filtered for $527 \mathrm{~nm} \pm 5 \mathrm{~nm}$, read out by a $4.5 \mathrm{GHz}$ oscilloscope (Tektronix SCD5000). The temporal resolution of the photo-detector is limited by the $20 \mathrm{~m}$ long $50 \Omega$ cable (Heliax) to around $250 \mathrm{ps}$. The three detectors (calorimeter, diode, CCD) are absolutely calibrated in situ using a low energy (20 J) $1 \mathrm{~ns}$ square laser pulse at $2 \omega$ with no target at tcc. We use spectrally calibrated filters to increase the dynamic range of the detectors as the transmitted power is changing for different target/beam configurations.

We use a disposable 3" BK7-glass plate $(1 \mathrm{~mm}$ thickness) as blast shield to prevent the coating of the TBD mirror with debris from the nearby target. The $8 \%$ baresurface reflection from the uncoated blast-shield with a diameter of $32 \mathrm{~cm}$ at the chamber wall is diverted from the TBD detector port by mounting it at a small angle relative to the TBD mirror $\left(7^{\circ}\right)$. The arm needs to be retracted and re-inserted after every laser shot in order to replace the blast shield in front of the mirror. As previous experiments have shown, the insertion procedure maintains alignment of the optical mirror within a fraction of a degree.

The maximum tolerable transmitted energy without beam spray ( $\mathrm{f} / 6.7$ cone) is around $50 \mathrm{~J}$ due to the $5 \mathrm{~J} / \mathrm{cm}^{2}$ damage threshold of the AR coating on the backside of the collection mirror (the damage threshold for the fused silica front side is i $20 \mathrm{~J} / \mathrm{cm}^{2}$ ).

In a slightly different configuration the TBD reflector arm was also used to calibrate the existing $2 \omega$ nearbackscattering imager (NBI) that is used to measure the fraction of backscattered light that is outside the collection lens of the full-aperture backscatter diagnostics (FABS) $[3,6]$. The NBI consists of two time integrating CCD cameras spectrally filtered for stimulated Brillouin scattering (SBS) and stimulated Raman scattering (SRS) from the $2 \omega$ beam. The cameras view the chamber wall surrounding the $2 \omega$ interaction beam port from the opposite side of the chamber. For an absolute calibration a high reflective $2 \omega$ convex mirror $(\mathrm{R}=60 \mathrm{~cm})$ is mounted in front of the TBD mirror at an angle of $13^{\circ}$. This mirror retro-reflects the light from a low energy calibration shot back to the interaction beam port (P9) and illuminates the chamber wall homogeneously over an area with a diameter of $50 \mathrm{~cm}$ (about twice the diameter of the port).

\section{PERFORMANCE OF THE DIAGNOSTICS}

First measurements were performed on hydrocarbongasbag targets [6]. Heating the target with an energy of $10 \mathrm{~kJ}$ produces a large-scale length plasma with an electron density and temperature around $5.5 \times 10^{20} \mathrm{~cm}^{-3}$ and 1.8-2 keV respectively. Using the TBD we measured 
the transmission of the interaction beam through the $~$ $2 \mathrm{~mm}$ long plasma [7]. The $1 \mathrm{~ns}$ long beam was spatially smoothed with a distributed phase plate that produced a vacuum spot diameter of about $200 \mu \mathrm{m}$ and an intensity of up to $10^{15} \mathrm{~W} / \mathrm{cm}^{2}$. Figure 3 shows the near-field
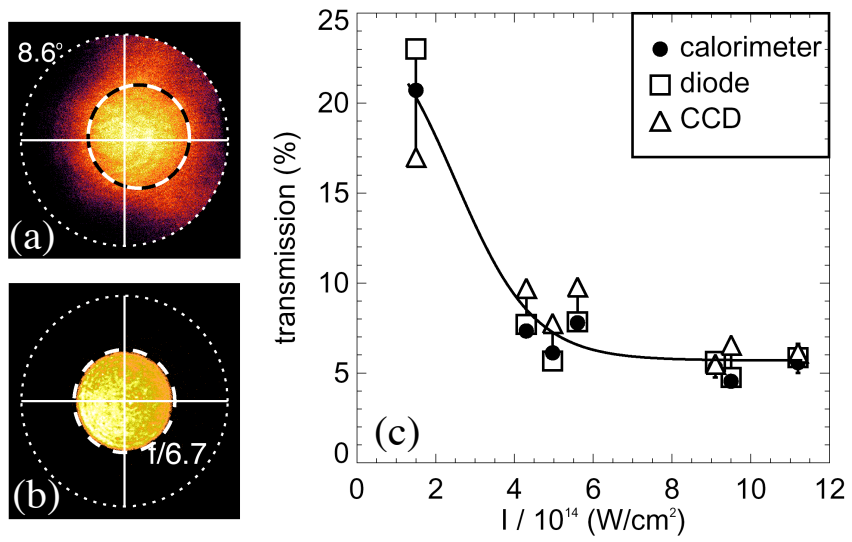

FIG. 3: (a) Time integrated near-field images of a high intensity $\left(10^{15} \mathrm{~W} / \mathrm{cm}^{2}\right)$ target shot (a) and a low energy calibration shot with no target (b). Beam transmission as a function of interaction beam intensity as measured with the different detectors (c).

images of the interaction beam at an intensity of $10^{15}$ $\mathrm{W} / \mathrm{cm}^{2}$ in the gasbag-plasma (a) and the low energy calibration shot without target (b). At the high intensity (a), the beam exhibits a large spray outside the initial $\mathrm{f} / 6.7$ cone (dashed inner circle) reaching the edge of the TBD collection mirror (dotted outer circle). Without a plasma (b) the beam is confined to the original $\mathrm{f} / 6.7$ cone. An analysis of the beam spray in (a) shows that only a third of the energy of the transmitted beam is contained inside the original $\mathrm{f} / 6.7$ cone. The absolute beam transmission was measured (Fig. 3c) independently with the calorimeter (circles), the photo-diode (squares) and the CCD camera (triangles). The signal to noise ratio is $~$ 1000:1, 200:1 and 40:1 for the calorimeter, the diode and the CCD camera, respectively, depending on the absolute beam transmission and the filter settings. All three detectors show similar results. For shots with intensities above $4 \cdot 10^{14} \mathrm{~W} / \mathrm{cm}^{2}$ the calorimeter and the diode measure the same absolute transmission within $5 \%$ (better than $1 \%$ in incident power). The discrepancy with the CCD camera data is larger $(20 \%)$ due to the poor signal to noise ratio of the camera. The large error at the low intensity shot is due to a reduced contrast on all 3 detectors.

\section{DETECTOR UPGRADE FOR SPECTRAL MEASUREMENTS}

A near-term upgrade to the detector will add spectral resolution to measure stimulated forward Raman scat- tering (SFRS) in the spectral range between $600 \mathrm{~nm}$ and $900 \mathrm{~nm}$ and stimulated forward Brillouin scattering (SFBS) at $\sim 527 \mathrm{~nm}$. SFBS light from the diffuser will be collected by a $400 \mu \mathrm{m}$ graded-index fiber (Mitsubishi SGLH400E) and sent to an optical spectrometer coupled to an S-20 streak camera. We will use a $1 / 2$ $\mathrm{m}$ SPEX spectrometer with a 1200 lines/mm grating in the second order and an entrance slit of $200 \mu \mathrm{m}$ with a spectral and temporal resolution of $0.5 \AA$ and 50 ps respectively $[3,6]$. SFRS light is transmitted through the first high-intensity $2 \omega$ turning mirror and is coupled via a second optical fiber to a SPEX 1/4 m spectrometer and a streak camera with an S-1 cathode. The SFRS system will consist of a low dispersion 150 lines $/ \mathrm{mm}$ grating yielding a spectral and temporal resolution of $1 \mathrm{~nm}$ and 50 ps respectively. Both the SFBS and SFRS spectrometers will be used simultaneously with the $2 \omega$ FABS [3], coupling two optical fibers in each spectrometer with a small time delay between the two signals. A fast, absolutely calibrated photo-detector with optical long-pass and short-pass filters will be used to measure the total SFRS energy in different spectral ranges.

\section{CONCLUSION}

We have implemented a $2 \omega$ transmitted beam diagnostic at the Omega laser facility. The TBD measures absolute transmitted beam power with an accuracy better than $1 \%$ in incident power and time integrated near-field images over twice the initial half-cone angle of the probe beam. We have demonstrated that the instrument can be absolutely calibrated in situ using a low energy laser pulse. The TBD was used to study the propagation of a $0.527 \mu \mathrm{m}$ laser beam through ignition-scale length plasmas. The performance of the instrument has satisfied the design requirements. A near term upgrade to the diagnostic will add spectral resolution and the possibility to measure stimulated forward Raman- and Brillouin scattering.

This work was performed under the auspices of the U.S. Department of Energy by the University of California Lawrence Livermore National Laboratory under contract No. W-7405-ENG-48. 
[1] N.D. Delamater et al., Phys. Plasmas 3(5), 2022 (1996)

[2] R.L. Kauffman et al., Phys. Plasmas, 5(5), 1927 (1998)

[3] A.J. Mackinnon et al., Rev. Sci. Instrum., to be published

[4] J.M. Sources et al., Fusion Technology, 30, 492 (1996)

[5] J.D. Moody et al., Rev. Sci. Instrum. 68(4), 1725 (1997)
[6] J.D. Moody et al., proceedings: $3^{\text {rd }}$ Interntional Conference on Inertial Fusion Sciences and Applications, Monterey, CA, Sep. 7-12 (2003)

[7] C. Niemann et al., submitted to Phys. Rev. Lett. 